\title{
Neonatal mortality rate (NMR) in intensive care unit (ICU) of Ardabil city hospitals and related factors
}

\author{
Mehrdad Mirzarahimi $^{1}$, Afsaneh Enteshari-Moghaddam ${ }^{2} *$, Sepideh Khademi $^{3}$
}

\begin{abstract}
${ }^{1}$ Department of Pediatrics, ${ }^{2}$ Department of Internal Medicine, ${ }^{3}$ General Practitioner, Faculty of Medicine, Ardabil University of Medical Science, Ardabil, Iran
\end{abstract}

Received: 08 August 2018

Accepted: 01 September 2018

\section{*Correspondence:}

Dr. Afsaneh Enteshari-Moghaddam,

E-mail: afsanehenteshary@gmail.com

Copyright: () the author(s), publisher and licensee Medip Academy. This is an open-access article distributed under the terms of the Creative Commons Attribution Non-Commercial License, which permits unrestricted non-commercial use, distribution, and reproduction in any medium, provided the original work is properly cited.

\begin{abstract}
Background: Neonatal mortality rate (NMR) is one of the important parameters in assessing quality of life in societies. NMR are often used as a standard index for evaluation health, education and social systems, nutritional status and health programs for neonates in countries and the first step in reducing mortality rate and promoting the level of this indicator is to identify the causes of mortality. Therefore, this study was conducted to determine the NMR in new born infants admitted to the ICU of Ardabil city hospitals.

Methods: This study was a descriptive cross-sectional study that has been done on 184 dead neonates in Ardabil city hospital during 2016-2017. The necessary information was extracted from the hospital records of neonates and entered in a provided checklist and then analyzed by statistical methods such as tables in SPSS version 19.

Results: The mortality rate of hospitalized infants in this research was $6.6 \%$. Of all patients, in $65.2 \%$ CBC were impaired and $58.7 \%$ of patients had impaired serologic and biochemical tests. $68.5 \%$ of patients had prematurity, $62.5 \%$ RDS and $20.7 \%$ had congenital anomalies.

Conclusions: Prematurity, RDS and congenital anomalies are the most important causes of NMR and we should programing to decreasing the NMR rate in future.
\end{abstract}

Keywords: Ardabil, ICU, Neonatal mortality rate, Prematurity

\section{INTRODUCTION}

Access to primary health care (PHC) is the right of every human and preventing of the death in children and mothers is one of the pillars of social justice. Provide maintenance and promotion of mothers and children health levels as vulnerable groups has a special place in the health services. Neonatal period as mean as the first 28 days after birth which was the stage of developing many physiological adaptations for ectopic life, is a vulnerable period and the high NMR is due to the high vulnerability of this time. ${ }^{1}$

Yearly near three million babies die in the whole world and $99 \%$ of them occur in the countries with low to middle economy but despite the great importance this health topic hasn't more attention. Also, yearly about 7.6 million children die due to preventable reasons that of them $40 \%$ occur in the infancy. ${ }^{2,3}$

NMR are often used as a standard index for evaluation health, education and social systems, nutritional status and health programs for neonates in countries and the first step in reducing mortality rate and promoting the level of this indicator is to identify the causes of mortality. The causes of neonatal mortality are generally classified into biological and non-biological groups. Although biological factors such as prematurity, infections and asphyxia at birth are known causes of neonatal mortality, non-biological causes are equally 
important, including socioeconomic condition, gender and mothers' education. Maternal and fetal prenatal issues and also conditions during childbirth, affect the perinatal mortality rate. Perinatal deaths are associated with issues such as intrauterine growth restriction, embryonic asphyxiation conditions such as fetal immaturity, severe congenital malformations, early and risky infection for neonate, low birth weight, early birth and congenital fatal abnormalities. ${ }^{4}$

Child death rate for children under five year in the country was 22.52 per 1,000 births and NMR is 15.29 per 1000 live births which accounts for $46 \%$ of the death for children under 5 years. ${ }^{5}$ In the study of Fallahzadeh et al, the most important causes of neonatal mortality were prematurity, congenital anomalies and respiratory distress; respectively and there was a significant relationship between birth time, gestational age and postpartum age with mortality rate. ${ }^{6}$ In Sankaran study, neonatal mortality rate was $4 \%$ and the most important factors were gestational age less than 24 weeks, gestational age between 24-28 weeks, congenital anomalies , infection and encephalopathy. ${ }^{4}$ In the study of Tariq, the NMR was $9 \%$ and neonatal infections and asphyxia during labor were two major causes of mortality. Respiratory distress syndrome, meconium aspiration and congenital anomalies are another important causes of neonatal mortality. $69 \%$ of deaths occur in low birth weight (LBW) infants and $74 \%$ in premature infants which indicating a high prevalence of mortality among LBW and premature infants. ${ }^{5}$ The aim of this study was to determine the NMR in ICU of Ardabil city hospitals and related factors.

\section{METHODS}

Study design: In this descriptive cross-sectional study, 184 dead neonates in ICU of Ardabil city hospitals were examined. By referring to the hospital records of the newborns, the necessary information such as prematurity and its related factors, infection, congenital anomalies and also demographic information including sex, weight, age at death time, mother's age, parity, history of disease in mother, type of delivery, gestational age in birth time were extracted and entered in a checklist.

\section{Inclusion criteria}

Neonates who had death during 28 day after birth entered in the study.

\section{Exclusion criteria}

Other newborns excluded from the study.

\section{Statistical analysis}

Collected data analyzed by statistical methods such as table in SPSS version 19.

\section{RESULTS}

In this study, the NMR was seen in 184 infants (6.6\%) which was occurred among 2774 registered birth during 2012-2013. $76(41.3 \%)$ of dead neonates were girl and rest were boy. Of them 109 (59.2\%) had weight low than $1500 \mathrm{gm}$. Of all dead neonates $144(78.3 \%)$ were born in gestational age less than 37 weeks. 33(17.95) of neonates died in the first day of life (Table 1).

Table 1: Demographic information of dead neonates.

\begin{tabular}{|c|c|c|}
\hline Variables & n & $\%$ \\
\hline Sox Male & 76 & 41.3 \\
\hline Sex Female & 108 & 58.7 \\
\hline \multicolumn{3}{|l|}{ Weight } \\
\hline$<1500$ & 109 & 59.2 \\
\hline $1500-2500$ & 32 & 17.4 \\
\hline$>2500$ & 43 & 23.4 \\
\hline \multicolumn{3}{|c|}{ Gestational age } \\
\hline$\leq 37$ & 144 & 78.3 \\
\hline $38-42$ & 37 & 20.1 \\
\hline$>42$ & 3 & 1.6 \\
\hline \multicolumn{3}{|c|}{ Age of neonate in time of death } \\
\hline First day & 33 & 17.9 \\
\hline 2-7 days & 89 & 48.4 \\
\hline $8-28$ days & 62 & 33.7 \\
\hline \multicolumn{3}{|c|}{ Residence place } \\
\hline Urban & 139 & 75.5 \\
\hline Rural & 45 & 24.5 \\
\hline \multicolumn{3}{|l|}{ Parity } \\
\hline 1 & 102 & 55.4 \\
\hline $2-4$ & 79 & 42.9 \\
\hline$\geq 5$ & 3 & 1.6 \\
\hline
\end{tabular}

$86(42.7 \%)$ of mothers had history of heart diseases. $37(20.1 \%)$ of them suffered to diseases such as GDM, Pre-eclampsia/eclampsia and other diseases during pregnancy time. Of all neonates' mothers, 17(9.2\%) had familial marriage. $3(1.6 \%)$ had history of smoking use. $105(57.1 \%)$ had nulipar and rest had multipar. $31 \%$ of neonates born by vaginal delivery and $69 \%$ born by caesarian section delivery. Of mothers $14.7 \%$ had positive history of $\mathrm{C} / \mathrm{S}$ (Table 2).

Table 2: Demographic data of mothers of dead neonates.

\begin{tabular}{|lll|}
\hline Variables & n & $\%$ \\
\hline History of hearth diseases & 86 & 46.7 \\
\hline Mother illness during pregnancy & 37 & 20.1 \\
\hline Positive familial of parents & 17 & 9.2 \\
\hline History of smoking use in mother & 3 & 1.6 \\
\hline Nulipar & 105 & 57.1 \\
\hline Multipar & 79 & 42.9 \\
\hline Vaginal delivery & 57 & 31 \\
\hline Cesarean delivery & 127 & 69 \\
\hline History of cesarean & 27 & 14.7 \\
\hline
\end{tabular}


After clinical examination of neonates, Lung diseases and Cyanosis with $84.2 \%$ and $52.7 \%$ were the most clinical symptom among neonates, respectively. Of dead neonates, $6(3.3 \%)$ had seizure and $12.1 \%$ had fever (Table 3).

Table 3: Clinical finding of dead neonates.

\begin{tabular}{|lll|}
\hline Symptoms & $\mathrm{n}$ & $\%$ \\
\hline Fever & 21 & 12.1 \\
\hline Jaundice & 18 & 9.7 \\
\hline Seizure & 6 & 3.3 \\
\hline Cyanosis & 97 & 52.7 \\
\hline Heart diseases & 25 & 13.6 \\
\hline Lung diseases & 155 & 84.2 \\
\hline Pneumothorax & 18 & 9.8 \\
\hline Disseminated intravascular coagulation & 23 & 12.5 \\
\hline
\end{tabular}

In summary 152 neonates use ventilator and in average they use ventilator for $3.6 \pm 3.5$ days. In Para clinical section, 120 dead neonates $(65.2 \%)$ had abnormal CBC and $108(58.7 \%)$ had Abnormal serology and biochemistry tests (Table 4).

Table 4: Para clinical finding in died neonates.

\begin{tabular}{|lll|}
\hline Para clinic & $\mathrm{n}$ & $\%$ \\
\hline Abnormal CBC & 120 & 65.2 \\
\hline $\begin{array}{l}\text { Abnormal serology and } \\
\text { biochemistry tests }\end{array}$ & 108 & 58.7 \\
\hline Positive CSF culture & 7 & 3.8 \\
\hline Positive urea culture & 12 & 6.5 \\
\hline Positive blood culture & 14 & 7.6 \\
\hline Abnormal CX-ray & 70 & 38 \\
\hline Other abnormal cases & 35 & 19 \\
\hline
\end{tabular}

Of all neonates, 38 neonates $(20.7 \%)$ had Congenital anomaly and $126(68.5 \%)$ had prematurity. Among neonates with congenital anomaly that of them 20 neonates were girl and 30 neonates were from urban area. Of all mothers 22 cases had history of heart disease. 8 parents of neonates had familial marriage. 7 neonates (3.8\%) had pneumonia caused by meconium aspiration that of them 5 neonates were girls. Of all neonates, 115 $(62.5 \%)$ had RDS that of them 7 neonates had familial marriage and 80 neonates' birth by $\mathrm{C} / \mathrm{S}$ and 89 neonates had weight lower than $1500 \mathrm{gm}$ (Table 5).

Table 5: Causes of death in neonates.

\begin{tabular}{|lll|}
\hline Causes & n & $\%$ \\
\hline Congenital anomaly & 38 & 20.7 \\
\hline Prematurity & 126 & 68.5 \\
\hline Infection & 33 & 17.9 \\
\hline Childbirth asphyxia & 13 & 7.1 \\
\hline Pneumonia caused by meconium aspiration & 7 & 3.8 \\
\hline RDS & 115 & 62.5 \\
\hline IVH & 6 & 3.3 \\
\hline Metabolic disease & 12 & 6.5 \\
\hline
\end{tabular}

\section{DISCUSSION}

In this study, the NMR was $6.6 \%$ of all births that is lower than the study of Pakistan with $9 \%$ and Canada with $4 \%$ which probably due to the difference in the level of health development in these communities.,

In this study, $41.3 \%$ of neonates were female and $58.7 \%$ were boy which was in line with other studies. There was no significant relationship between gender and causes of death in these studies. ${ }^{7-9}$

Of all infants, $141(76.6 \%)$ had LBW which was higher than China and Fonsca's study and less than the study of India with $90 \%$ and the highest frequency was observed in neonates weighing less than 1000 gr of all neonates, $144(78.3 \%)$ were preterm which was similar to MirzaRahimi study with $84 \%$ and Tariq study with $74 \%$ and was more than Stoil study with $38.6 \%$. $^{1,5,7-9}$

$17.9 \%$ of the newborns died on the first day and $48.4 \%$ of the newborns died in the first week of birth that this result less than the results of Mirza Rahimi's study with $87.7 \%$ during the first week and also the study of Fallahzadeh and Nayerri study which $95 \%$ and $78 \%$ of deaths occurred during the first week, respectively. $55.4 \%$ of infants had the first parity and studies have shown that pregnancy complications are more prevalent in first born younger women and this can be related to their lower awareness about pregnancy problems and care during pregnancy and after pregnancy. ${ }^{6,9-11} 20.1 \%$ of the mothers had pregnancy complications that this amount was less than the result of Taheri et al study with $34 \% .^{12}$

In this study, $61 \%$ of neonates were born with cesarean section and $14 \%$ had a history of cesarean section; cesarean section neonate issues probably related to the long-term maternal anesthesia and special surgical conditions. Because anesthetics and analgesics cause hypoxia and a mild respiratory depression in the baby and as a result, when not be careful in the sue of anesthetic drugs or their effects adds to adverse conditions such as prematurity, asphyxia and trauma it will be accompanied with neonate side effects. ${ }^{13}$

In this study, the prevalence of clinical findings among neonates was respectively lung problem, scyanosis, heart disease, DIC and fever. In the study of Taheri et al and Pour Arian et al the most common cause was respiratory distress syndrome and prematurity and pointed to the prematurity as one of the most important factors that affected infant mortality. In this study, the causes of death were prematurity, RDS, congenital anomalies, infection, asphyxiation, metabolic disease, pneumonia caused by meconium aspiration and IVH. In the study of Taheri et al, the most common cause of neonate death were respiratory distress and prematurity. ${ }^{12,14}$ Shirvani in his study by the title "causes of neonatal mortality" were reported prematurity and LBW as a major cause of death. ${ }^{15}$ In the study of Rakholia et al, the main causes of 
mortality included $25.6 \%$ prematurity, $21.6 \%$ sepsis, $19.5 \%$ asphyxia and $17.3 \%$ RDS. ${ }^{16}$ In the study by Mungyeh, the main cause of death was sepsis $(37.85 \%)$, prematurity $(31.26 \%)$, delivery asphyxia (16\%) and congenital anomalies $(10.54 \%){ }^{17}$

In the study of Abdellatif, the primary reason of neonatal death was prematurity and its complications (52\%), deadly congenital malformations (29\%), another proprietary diagnoses (12\%) and neonates asphyxia (7\%). ${ }^{18}$ In the study of Aramesh, the most common causes of mortality were prematurity and its complications $(58.8 \%)$, congenital anomalies (17.3\%), asphyxia around birth (8\%), infection (6\%) and other reasons $(9 / 9 \%){ }^{19}$

In Nouri study, the most common causes of death were respectively prematurity $(47.42 \%)$, congenital anomalies $(22.42 \%)$ and accidents $(9.79 \%)$ and $82.73 \%$ of dead neonates had weigh below $2500 \mathrm{~g}$ and $60 \%$ of death due to prematurity was in first gravid mothers. ${ }^{20}$ In the study of Javanmardi, the most common causes of neonatal death in NICU hospitals were prematurity (44.6\%), congenital anomalies $(23.5 \%)$ and RDS $(11.6 \%)$ and in non NICU hospitals were prematurity (42.6\%), congenital anomalies (21.3\%) and RDS (12.7\%) and in other hospitals were prematurely (47.5\%), RDS (18.1\%) and congenital anomalies $(12.4 \%)$, respectively. ${ }^{21}$

In the study of Bijari et al, the most common causes of death were prematurity $(36.4 \%)$, birth asphyxia (20.7\%), sepsis $(13.6 \%)$ and congenital abnormalities $(13.8 \%)$ and the unknown reasons $(15.3 \%){ }^{22}$

\section{CONCLUSION}

Results showed that the prematurity, RDS and congenital malformations are the three main causes of NMR. Due to the high prevalence of neonatal deaths under 37 weeks, more care in preventing preterm labor as the most important cause of neonatal mortality has particular importance in reducing NMR. Improved use of special care, optimal use of ventilator, fluid therapy and full intravenous nutrition, prevention of hospital infection can help reduce mortality in preterm infants. To control the prevalence of congenital diseases in the community counselling pre-marital before and during pregnancy and detection patients in families with a history of previous genetic diseases can be many helpful. Increased prenatal care, monitoring the condition of the mother and the fetus and timely selection of the proper method for termination of pregnancy can reduce the damage to the baby during pregnancy. It is also necessary to have a more regular documentation of hospital records and complete registration of mother's maternity records in a new born case so that the neonatal mortality monitoring system is more accurate. Health centers should be provided a more comprehensive, high quality system for periodic pregnancy monitoring. By identifying and early intervening in high risk pregnancies, it reduces the risk of perinatal complications and mortality.

Funding: No funding sources

Conflict of interest: None declared

Ethical approval: The study was approved by the Institutional Ethics Committee

\section{REFERENCES}

1. Feigelman S. Assessment of fetal growth and development. In: Kliegman RM, Stanton BF, St Geme JW, Schor NF, eds. Nelson text book of Pediatrics, $20^{\text {th }}$ ed. Saunders, Philadelphia; 2016:6062.

2. Moss W, Darmstadt GL, Marsh DR, Black RE, Santosham M. Research priorities for the reduction of perinatal and neonatal morbidity and mortality in developing country communities. J Perinat Med. 2002;22:484-95.

3. $\mathrm{Yu}$ VY. Global, regional and national perinatal and neonatal mortality. J Perinat Med. 2003;31(5):376-9.

4. Sankaran K, Chien Li-Yin, Walker R, Seshia M, Ohlsson A, Lee Sh K. Variations in mortality rates among Canadian neonatal intensive care units. CMAJ. 2002;166(2):173-8.

5. Tariq P, Kundi Z. Determinants of neonatal mortality. J Pak Med Assoc. 1999;49(3):56-60.

6. Fallahzadeh H, Gholestan M, Rezvan A, Mohtashami A, Hakimi Kh, Ahmadabadi M. Study the neonaltal mortality rate and some related factors in Yazd city. J Shahid Sadoughi Univ Med Sci. 2000;8(4):54-7.

7. Chen ZL, Li FY. Mortality rate and causes of death of premature and low-birth-weight infants in cities. Zhonghua Fu Chan Ke Za Zhi. 1993;28(1):27-30.

8. Fonseka P, Wijewardene K, Harendra de Silva DG, Goonaratna C, Wijeyasiri WA. Neonatal and postneonatal mortality in the Galle district. Ceylon Med J. 1994;39(3):147-8.

9. Mirzarahimi M, Abedi A, Shahnazi F, Saadati H, Enteshari A. Causes and rate of mortality among the newborns in NICU and newborns unit at Imam Khomeini and Alavi Hospitals in Ardabil from September 2006 to September 2007. J Ardabil Univ Med Sci. 2008;8(4):424-30.

10. Nayerri F, Amini E, Eloumi Z, Dehghan-niri A. Study the main causes of NMR based on ICD in valiasr hospital. Iranian J Pediatr. 2007;17(10):22-6.

11. Baer L, Henry S. Analysis of 1600 thrombcytopenic neonates in a multi hospital health care system. J Perinatol. 2007;27(12):790-6.

12. Taheri F, Sharifzadeh G, Kaheni S, Saboori G. Epidemiology of Neonates Hospitalized in NICU of Vali e asr Hospital, Birjand, in 2005-2006. Mod Care J. 2007;4(1):29-34.

13. Sachdeva RC. Quality and safety in healthcare for children. In: Kliegman RM, Stanton BF, St Geme JW, Schor NF, eds. Nelson text book of Pediatrics, $20^{\text {th }}$ ed. Saunders, Philadelphia; 2016:18-26. 
14. Pour Arian SH, Sabet S. Study the effective causes on transition of neonates to ICU in Shiraz hospital. Shiraz Med Res J. 2005;1(3):34-42.

15. Shirvani F, Khosroshahi N. A survey of frequency and causes of perinatal mortality in Tehran, 1995. Tehran Univ Med J. 1998;56(1):69-73.

16. Rakholia R, Rawat V, Bano M, Singh G. Neonatal morbidity and mortality of sick newborns admitted in a teaching hospital of Uttarakhand. Chris Med J Health Res. 2014;1:228-34.

17. Mah Mungyeh E, Chiabi A, Tchokoteu Pouasse FL, Nguefack S, Bogne JB, Siyou H, et al . Neonatal mortality in a referral hospital in Cameroon over a seven year period: trends, associated factors and causes. African Health Sci. 2014;14(3):517-25.

18. Abdellatif $M$, Ahmed $M$, Flordeliz Bataclan $M$, Ahmed Khan A, Al Battashi A, Al Maniri A. The patterns and causes of neonatal mortality at a tertiary hospital in Oman. Oman Med J. 2013;28(6):422-6.

19. Aramesh M, Malekian A, Dehdashtian M, Shahvari A, Monjezi L. Determine the rate and cause of NMR in ICU of Ahvaz hospital in 2011. Razi Med J. 2004;21(120):36-43.

20. Nouri A, Barati L, Qhezelsofly F, Niazi S. Causes of infant mortality in Kalaleh city during 2004-2012. Jorjani Biomed J. 2013;1(2):32-7.

21. Javanmardi Z, Beigi Z, Ghodoosi A. Investigating about the causes of neonates' death in the hospitals of Isfahan Province. Ir J Forensic Med. 2010;15(4):229-33.

22. Bahman-Bijari B, Niknafs P, Maddahiyan S. Causes of neonatal mortality in Kerman province in 1387 (2008-2009). J Urmia Univ Med Sci. 2012;22(6):501-6.

Cite this article as: Mirzarahimi M, EnteshariMoghaddam A, Khademi S. Neonatal mortality rate (NMR) in intensive care unit (ICU) of Ardabil city hospitals and related factors. Int J Reprod Contracept Obstet Gynecol 2018;7:4531-5. 